\title{
NONCLASSICAL EIGENVALUE ASYMPTOTICS FOR OPERATORS OF SCHRÖDINGER TYPE
}

\section{DAVID GURARIE}

We consider operators in the form $A=-\nabla \cdot \rho \nabla+V(x)$ on $\mathbf{R}^{n}$, where metric $\rho=\left(\rho_{i j}(x)\right) \geq 0$ and potential $V(x) \geq 0$. The classical Weyl principle for asymptotic distribution of large eigenvalues of $A$ states that the counting function

$$
N(\lambda)=\#\left\{\lambda_{j} \leq \lambda\right\} \sim \operatorname{Vol}\{(x ; \xi) \mid \rho \xi \cdot \xi+V(x) \leq \lambda\} \quad \text { as } \lambda \rightarrow \infty .
$$

(See for instance $[\mathbf{G u}]$.) Integrating out variable $\xi$ we can rewrite it as

$$
N(\lambda) \sim \frac{\omega_{n}}{(2 \pi)^{n}} \int(\lambda-V)_{+}^{n / 2} \frac{d x}{\sqrt{\operatorname{det} \rho}} .
$$

If potential $V$ and metric $\rho$ are assumed to be homogeneous in $x, V(x)=$ $|x|^{\alpha} V\left(x^{\prime}\right) ; \rho_{i j}(x)=|x|^{\beta} \rho_{i j}\left(x^{\prime}\right), x^{\prime}=x /|x|$, then (1) reduces to

$$
N(\lambda) \sim C \lambda^{[n / 2+(1-\beta / 2) n / \alpha]} \int V^{-(n / \alpha)(1-\beta / 2)} \frac{d S}{\sqrt{\operatorname{det} \rho}} ;
$$

integration over the unit sphere $S$ with constant

$$
C=\frac{\omega_{n}}{(2 \pi)^{n} \alpha} B\left(\frac{n}{2}+1 ; \frac{n}{\alpha}(1-\beta / 2)\right),
$$

which depends on the volume $\omega_{n}$ of the unit sphere in $\mathbf{R}^{n}$ and the beta function.

Assuming $\beta<2$ we see that integral (2) becomes divergent if $V\left(x^{\prime}\right)$ vanishes to a sufficiently high order. The simplest such potential is $V(x, y)=|x|^{\alpha}|y|^{\beta}$ on $\mathbf{R}^{n}+\mathbf{R}^{m}$.

The Weyl (volume counting) principle, when applied to the corresponding Schrödinger operator $-\Delta+V(x)$, fails to predict discrete spectrum below any energy level $\lambda>0$. However, as was shown by D. Robert [Ro] and B. Simon [Si], $A$ has purely discrete spectrum $\left\{\lambda_{j}\right\} \rightarrow+\infty$ (for qualitative explanation of this phenomenon see $[\mathbf{F e}])$. Moreover, the "nonclassical" asymptotics of $N(\lambda)$ was derived for such $A$.

Recently M. Solomyak [So] studied a general class of Schrödinger operators $-\Delta+V(x)$ with homogeneous potentials $V$ subject to the following constraint:

(A) zeros of $V,\{x: V(x)=0\}$ form a smooth cone $\Sigma$ in $\mathbf{R}^{n}$ of dimension $m$, and $V$ vanishes on $\Sigma$ "uniformly" to order $b>0$.

Introducing variables $x \in \Sigma$ and $y \in N_{x}$ (the normal to $\Sigma$ at $\{x\}$ ), hypothesis (A) means that there exists

$$
\lim _{t \rightarrow 0} t^{-b} V(x+t y)=V_{0}(x, y) .
$$

Received by the editors November 18, 1985.

1980 Mathematics Subject Classification (1985 Revision). Primary 35P20; Secondary $35 \mathrm{~J} 10$. 
It is easy to see that $V_{0}(x, y)$ has mixed homogeneity

$$
V_{0}(x, y)=|x|^{a}|y|^{b} V_{0}\left(x^{\prime}, y^{\prime}\right) ; \quad a+b=\alpha
$$

and $\dot{V}_{0}$ approximates $V$ in a small conical neighborhood $\Sigma_{\varepsilon}$ of $\Sigma$ :

$$
\Sigma_{\varepsilon}=\{x+y|x \in \Sigma ;| y|<\varepsilon| x \mid\} .
$$

Under hypothesis (A) M. Solomyak [So] derived asymptotics of $N(\lambda)$ for such operators $A=-\Delta+V(x)$ in terms of eigenvalues $\left\{\lambda_{j}(x)\right\}_{1}^{\infty}$ of an auxiliary family of Schrödinger operators $\left\{L(x)=-\Delta_{y}+V_{0}(x, y)\right\}_{x \in \Sigma}$. Namely,

$$
N(\lambda) \sim C \lambda^{\frac{m}{2}\left(1+\frac{2+b}{a}\right)} \int_{\Sigma^{\prime}} \sum_{1}^{\infty} \lambda_{j}\left(x^{\prime}\right)^{-m(2+b) / 2 a} d S,
$$

the integral is over $\Sigma^{\prime}=\Sigma \cap S$ (unit sphere).

Notice that each operator $L(x)$ has "classical type," so Weyl's principle (2) applies to $\left\{\lambda_{j}(x)\right\}_{1}^{\infty}$,

$$
\#\left\{\lambda_{j}(x) \leq \lambda\right\} \sim c(x) \lambda^{(n-m)(1 / 2+1 / b)} .
$$

Let us also observe that a polynomial asymptotics of $N(x) \sim c \lambda^{p}$ implies convergence of the series

$$
\sum_{1}^{\infty} \lambda_{j}^{-q}<\infty, \text { with any } q>p .
$$

Hence by (5) the sum in (4) converges provided

$$
q=m(2+b) / 2 a>p=(n-m)(1 / 2+1 / b) .
$$

Condition (6) is sufficient for validity of (4). In the critical case $q=p$ an additional $\log \lambda$ factor appears in (4).

The method of [So] was based on the variational formulation of the problem and certain eigenvalue estimates for Schrödinger operators in conical regions obtained in [Ros].

In the present paper we shall outline a different approach based on pseudodifferential calculus with operator-valued symbols in the spirit of [Ro]. This method allows us to recover Solomyak's result (4) and to extend it in various directions, including operators of the form $-\nabla \cdot \rho \nabla+V(x)$.

We propose the following principle, which governs nonclassical asymptotics: the main contribution to $N(\lambda)$ comes from the degeneracy set $\Sigma$ (critical set) of $V$.

According to this principle we want to "localize" $A$ to a small (conical) neighborhood of $\Sigma$. Precisely, let us introduce the "model" operator

$$
A_{0}=-\Delta_{\Sigma}+L(x)=-\Delta_{\Sigma}+\left[-\Delta_{N}-2 \nabla_{x} \cdot \rho^{\prime} \nabla_{y}+V_{0}(x, y)\right]
$$

on the manifold $\mathcal{N}(\Sigma)=\bigcup_{x \in \Sigma} N_{x}$, normal bundle to $\Sigma$, where $\Delta_{\Sigma}, \Delta_{N}$ are the Laplace-Beltrami operators on $\Sigma$ and the normal space, $N=N_{x}$, with respect to the metrics induced by $\rho_{i j}$, and $\rho^{\prime}$ is the "off diagonal" part of $\rho$.

Writing $A=-\nabla \cdot \rho \nabla+V$ in normal coordinates $(x, y)$ one can show that $A=A_{0}+$ "small perturbation" in a conical neighborhood $\Sigma_{\varepsilon}$ of $\Sigma$. So we expect $N(\lambda ; A) \sim N\left(\lambda ; A_{0}\right)$, as $\lambda \rightarrow \infty$. 
To study the eigenvalue distribution one usually works with certain integral "transforms" of $N(\lambda)$, like $\operatorname{tr} e^{-t A}=\int^{+\infty} e^{-\lambda t} d N(\lambda)$ or $\operatorname{tr}(\varsigma+A)^{-l}=$ $\int^{+\infty}(\zeta+\lambda)^{-l} d N(\lambda)$.

We prefer to work with the latter. Once the asymptotics

$$
\operatorname{tr}(\zeta+A)^{-l} \sim c_{0} \varsigma^{-l+p} \text { as } \zeta \rightarrow \infty
$$

is established for $\operatorname{tr} R_{S}^{l}$ one can go back to the asymptotics of $N(\lambda) \sim c \lambda^{p}$, as $\lambda \rightarrow \infty$, by the Tauberian Theorem of M. V. Keldysh (see [Ro]). The relation between the two constants is $c=c_{0} / p B(p ; l-p)$.

So we need to establish (8).

Operator $A$ can be thought of as a differential operator on $\Sigma$ with operatorvalued symbol $\sum g^{i j} \xi_{i} \xi_{j}+L(x)$, where metric $g=\rho_{\Sigma}-\rho^{\prime *} \rho_{N}^{-1} \rho^{\prime}$ on $\Sigma$ is constructed from the tangent $\rho_{\Sigma}$ and normal $\rho_{N}$ components of $\rho$. Then the parametrix (approximate inverse) of $\left(\zeta+A_{0}\right)^{-l}$ can be constructed as an operator-valued $\Psi$ DO $K=K_{\varsigma}^{(l)}$ with symbol

$$
\sigma_{K}=\left[\varsigma+\sum g^{i j} \xi_{i} \xi_{j}+L(x)\right]^{-l} .
$$

According to our principle we want to localize kernels $R_{\zeta}^{l}=(\varsigma+A)^{-l}$; $\tilde{R}^{l}=\left(\zeta+A_{0}\right)^{-l}$ and $K_{\zeta}^{(l)}$ to a small conical neighborhood $\Sigma_{\varepsilon}$ of $\Sigma$. Let us introduce a cut-off function

$$
\chi_{\varepsilon}= \begin{cases}1 & \text { on } \Sigma_{\varepsilon} \\ 0 & \text { outside }\end{cases}
$$

and define an orthogonal projection $P_{\varepsilon} u=\chi_{\varepsilon} u$ from $L^{2}\left(\mathbf{R}^{n}\right)$ onto $L^{2}\left(\Sigma_{\varepsilon}\right)$.

The following lemma plays the central role in the localization procedure.

LEMMA. All traces below are equivalent as $\zeta \rightarrow \infty$.

(i) $\operatorname{tr}(\varsigma+A)^{-l} \sim \operatorname{tr} P(\varsigma+A)^{-l} P$,

(ii) $\operatorname{tr}\left(\zeta+A_{0}\right)^{-l} \sim \operatorname{tr} P\left(\varsigma+A_{0}\right)^{-l} P$,

(iii) $\operatorname{tr} K_{\varsigma}^{(l)} \sim \operatorname{tr} P K_{\varsigma}^{(l)} P$,

(iv) traces of "truncated" operators : $P(\varsigma+A)^{-l} P, P\left(\varsigma+A_{0}\right)^{-l} P$, and $P K_{\zeta}^{(l)} P$ are all equivalent.

From the lemma follows

$$
\operatorname{tr}(\zeta+A)^{-l} \sim \operatorname{tr} K^{(l)} \text { as } \zeta \rightarrow \infty .
$$

Now it remains to compute the trace of an operator-valued $\psi \mathrm{DO} K_{\varsigma}^{(l)}$

$$
\operatorname{tr} K_{\zeta}^{(l)}=\iint \sum_{k=1}^{\infty}\left[\varsigma+\sum g^{i j} \xi_{i} \xi_{j}+\lambda_{k}(x)\right]^{-l} d \xi d x .
$$

Integrating out variables $\xi$, using homogeneity of $\lambda_{j}(x)$ and $\rho(x)$, and introducing "polar coordinates" on $\Sigma$ to reduce integration over the cone $\Sigma$ to a subset $\Sigma^{\prime}=\Sigma \cap S$, we get

$$
\operatorname{tr} K_{\zeta}^{(l)}=C_{0 \zeta^{-l+m(1 / 2+\theta)}} \int_{\Sigma} \sum_{1}^{\infty} \lambda_{j}\left(x^{\prime}\right)^{-m \theta} \frac{d x^{\prime}}{\sqrt{\operatorname{det} g^{i j}\left(x^{\prime}\right)}}
$$


with constants

(12) $s=\frac{\beta b+2 a}{2+b} ; \quad \theta=\frac{1}{s}(1-\beta / 2) ; \quad C_{0}=\int_{0}^{\infty} r^{m(1-\beta / 2)}\left(1-r^{s}\right)^{m / 2-l} d r$.

Remembering that $\left\{\lambda_{j}\left(x^{\prime}\right)\right\}$ obey the classical asymptotics (5) with exponent $p=(n-m)(2+b) / 2 b$, we obtain a sufficient condition of convergence of series (11)

$$
m \theta=\frac{m}{s}(1-\beta / 2)>p=\frac{(n-m)(2+b)}{2 b} \text { or } \frac{m(2-\beta)}{b+2 a}>\frac{n-m}{b} .
$$

Thus we have established the following

THEOREM. If operator $A=-\nabla \cdot \rho \nabla+V$ with homogeneous potential $V(x)=|x|^{\alpha} V\left(x^{\prime}\right) \geq 0$ and nondegenerate metric $\rho_{i j}(x)=|x|^{\beta} \rho_{i j}\left(x^{\prime}\right)>0$ satisfies hypothesis $(\bar{A})$, then spectral function $N(\lambda)$ of $A$ admits the nonclassical asymptotics

$$
N(\lambda) \sim C \lambda^{m(1 / 2+\theta)} \int_{\Sigma^{\prime}} \sum_{1}^{\infty} \lambda_{j}\left(x^{\prime}\right)^{-m \theta} \frac{d x^{\prime}}{\sqrt{\operatorname{det} g^{i j}\left(x^{\prime}\right)}},
$$

provided sufficient condition (13) holds. The metric $\left(g^{i j}\right)$ on $\Sigma$ is obtained from components of metric $\rho$.

REMARKS. (i) Formula (14) includes both the classical formula (2) with $\beta=0$ and $s=a$ (i.e., $b=0$ ) and all previously studied nonclassical asymptotics [Ro, Si, So] (the latter corresponds to $\beta=0$ ).

(ii) In the critical case (equality $m \theta=p$ in (13)) an additional $\log \lambda$ factor appears in (16). The argument requires some modification: Before passing to the limit in the sum $\sum_{1}^{\infty} \lambda_{j}^{-m \theta}$ and integration over $\Sigma$ one has to "localize" $K_{\zeta}^{(l)}$ to a compact region in $\Sigma$.

We shall illustrate our theorem and conditions by the following

EXAMPLE. Take scalar metric $\left(\rho_{i j}\right)=\rho=\left(t^{2}+|x|^{2}\right)^{\beta / 2} I_{n \times n}$ and potential $V=\left(t^{2}-|x|^{2}\right)^{\beta / 2}$ in the space $\mathbf{R}^{n}=\left\{(t, x): t \in \mathbf{R} ; x \in \mathbf{R}^{n-1}\right\}$. The degeneracy set of $V$ is the standard cone $\Sigma=\{(t, x): t= \pm|x|\}$ in $\mathbf{R}^{n}$.

Direct calculation shows: $a=b=\alpha / 2$ and $V_{0}(x, y)=|x|^{\alpha / 2}|y|^{\alpha / 2}$.

Condition (15) for convergence of the series of eigenvalues $\sum_{j} \lambda_{j}^{-(n-1) \theta}$ of the operator $L(x)=-d^{2} / d y^{2}+|y|^{\alpha / 2}$ on $\mathbf{R}$ becomes

$$
\frac{\beta+2}{2-\beta}<n-1 \text { or } \beta<\frac{2(n-2)}{n},
$$

and the eigenvalue asymptotics takes a form

$$
N(\lambda) \sim C \lambda^{(n-1)(1 / 2+\theta)} \sum_{1}^{\infty} \lambda_{j}^{-(n-1) \theta} \quad \text { with } \theta=\frac{(4+\alpha)(2-\beta)}{2 \alpha(\beta+2)} .
$$

\section{REFERENCES}

[Fe] C. Fefferman, The uncertainty principle, Bull. Amer. Math. Soc. N.S. 9 (1983), 129-206.

[Gu] D. Gurarie, $L^{p}$ and spectral theory for a class of global elliptic operators, preprint. 
[Ro] D. Robert, Comportement asymptotique des valeurs propres d'opérateurs du type Schrödinger à potentiel "dégénérê", J. Math. Pures Appl. 61 (1982), 275-300.

[Ros] G. V. Rosenblum, Distribution of eigenvalues of singular differential operators, Izv. Vyssh. Uchebn. Zaved. 1 (1976), 75-86. (Russian)

[Si] B. Simon, Nonclassical eigenvalue asymptotics, J. Funct. Anal. 53 (1983), 84-98.

[So] M. Z. Solomyak, Spectral asymptotics of Schrödinger operators with non-regular homogeneous potential, Mat. Sb. 127 (1985), 21-39. (Russian)

Department of Mathematics and Statistics, Case Western Reserve UNIVERSITY, CleVELAND, OHIO 44106 
